\title{
Las neuronas espejo y el desarrollo de la empatía frente a la agresión y el conflicto en la escuela ${ }^{1}$
}

\author{
Rosa Isabel Galvis² \\ Corporación Universitaria Minuto de Dios, Bogotá, Colombia \\ galvisrosaisabel@gmail.com
}

1 Artículo de investigación derivado del trabajo de investigación sobre la prevención del cyberbullying, desarrollado en la Maestría en Psicología, Universidad de Los Andes.

2 Docente-investigadora, Facultad de Educación, Uniminuto, Bogotá. 
Tema Libre

\title{
Las neuronas espejo y el desarrollo de la empatía frente a la agresión y el conflicto en la escuela
}

\section{Resumen}

El presente trabajo está orientado al estudio y análisis teórico de la empatía, su desarrollo y el papel que desempeña en los procesos de interacción social, con miras a precisar aspectos de carácter neurobiológico, psicosocia y cultural que puedan incidir en los procesos de formación. A la fecha se ha encontrado que la empatía es uno de los aspectos psicosociales que desempeña un papel importante en la resolución de situaciones de conflicto y agresión en los entornos escolares; por lo tanto, el reflexionar en torno a los diferentes aspectos de carácter teórico y metodológico que aporten a su fortalecimiento y contribuyan a la formación de individuos empáticos y sensibles respecto al dolor de otros, podría plantearse como una alternativa para enfrentar situaciones de conflicto y agresión presentes actualmente de manera constante en los entornos escolares, y motivar así el diseño para e futuro, de posibles alternativas pedagógicas que enfrenten este problema.

Palabras clave: empatía, interacción social, neuronas espejo, conflicto escolar.

\section{Symmetrical Neurons and the development of empathy against aggression and conflict in the school}

\begin{abstract}
This work aimed at studying and theoretical analysis of empathy, its development and role in the processes of social interaction, in order to clarify aspects of neurobiological, psychosocial and cultural nature that may affect the formation processes. So far, we have found that empathy is one of the psychosocial aspects that play an important role in the resolution of conflict and aggression within the school environment. Therefore, this is a reflection on the various aspects of theoretical and methodological problems that contribute to strengthen and allow formation of empathetic and sensitive individuals that can feel the pain of others. This could be viewed as an alternative to deal with the actual and constant conflict and aggression present in school environments, and thus motivate a future design of potential pedagogical alternatives that would address this problem.
\end{abstract}

Keywords: empathy, social interaction, mirror neurons, school conflict 
Una de las problemáticas que hoy en día aqueja a la sociedad, y que está presente en los entornos escolares, es la manifestación franca y abierta de comportamientos agresivos por parte de niños, niñas y adolescentes. Sea dicha agresión directa o indirecta, presencial o virtual (con apoyo de las tecnologías de la información y las comunicaciones), es un hecho que ha llevado a investigadores de ciencias humanas y sociales (psicólogos, pedagogos, sociólogos, educadores, biólogos y expertos en neurociencia) a buscar una explicación y posibles alternativas de solución a este fenómeno. Estos esfuerzos investigativos se han centrado en el estudio y análisis de aspectos psicosociales que fundamentan las interacciones sociales, entre los cuales el abordaje de la empatía es importante por la relación inversa entre ésta y la manifestación de comportamientos agresivos (Eisenberg y Strayer, 1992; Cepeda, 2004; Chaux, 2004; Cepeda, 2004; García, González y Maestú, 2011: Gutiérrez, Escartí y Pascual, 2011; Chaux, 2012). La relevancia de su estudio es tal, que no sólo ha sido objeto de análisis desde una perspectiva psicosocial (Eisenberg y Strayer, 1992; Feshbach, 1983; Salmivalli, 1996), sino que también ha sido abordada desde la neurociencia, con la aparición en el panorama científico de las neuronas espejo, descubrimiento a cargo de los investigadores Rizzolatti, Fadiga, Galese, Fogassi (1996), quienes han brindado un soporte neurobiológico de trascendencia para su comprensión.

En los últimos años se han venido desarrollando diversas investigaciones respecto a la influencia de este sistema de neuronas en el comportamiento social y moral de los seres humanos, haciendo énfasis en la incidencia de de dichas neuronas espejo especialmente en los comportamientos prosociales de la persona (Moya-Albiol, Herrero y Bernal, 2011; Deceaty (2014) respecto a la incidencia de las neuronas espejo en el desarrollo de fenómenos psicosociales como la empatía y la simpatía. Sin embargo, ofrece a la vez una postura crítica al respecto, cuando finalmente concluye que dicha influencia de estas neuronas en la repetición de conductas, está más asociada a comportamientos motores, restando influencia en procesos más complejos como lo son los comportamientos y actitudes sociales. En este sentido, se hace necesario ahondar en el estudio de este tema, principalmente en lo que respecta a estudios de base neuropsicológica, sin restar importancia frente a la necesidad de estudios de orden psicológico y social que aporten al fortalecimiento de la empatía.

\section{Perspectivas teóricas en torno al concepto de empatía}

El concepto de la empatía ha sido explicado por diferentes autores como la capacidad del individuo para "colocarse en el lugar del otro" de tal manera que le permita identificar sus sentimientos y emociones, hasta el punto de lograr experimentar lo mismo que éstos viven y experimentan. Desde los estudios realizados por (Lipps, 1903; Feshbach, 1983; Eisenberg y Strayer, 1992; Salmivalli, 1996; Chaux, 2004; Chaux, 2012; Gutiérrez, Escartí y Pascual, 2011) hasta los postulados más recientes propuestos por Deceaty (2014), la empatía ha sido considerada un aspecto fundamental para el 
desarrollo psicológico y emocional de los individuos, por cuanto es un factor que debe ser tenido en cuenta si se pretende propiciar ambientes de interacción social que ofrezcan a los individuos en formación entornos de armonía y sana convivencia. Para el presente trabajo, se retoma la conceptualización de empatía ofrecida por Gutiérrez et al. $(2011$, p.1) quienes la definen como "una respuesta afectiva de comprensión sobre el estado emocional de otros, que induce a sentir el estado en que se encuentra el otro". Al respecto, autores que han profundizado en su estudio, entre los que se encuentran Bandura y Hoffman citados por Gutiérrez et al. (2011) mencionan otro elemento que está involucrado en los procesos empáticos, relacionado con el componente cognitivo que implica un nivel de comprensión de esos estados afectivos de las otras personas, lo que ha llevado a considerar la empatía como un proceso multifuncional que involucra factores de índole emocional, cognitivo y social (Garaigordobil y García, 2006; Mestre et. al. 2002; González-Blasco, et al., 2013)

Con respecto a la relación que se ha encontrado entre las manifestaciones empáticas al interior de las interacciones sociales, Gutiérrez, et al. (2011) relacionan la empatía con la disposición por parte de las personas para realizar comportamientos prosociales, factor importante para enfrentar e inhibir la manifestación de comportamientos agresivos. En este sentido, el estudio desarrollado por Mestre, Samper y Frías (2002) demuestra el papel que desempeña la empatía como función moduladora facilitando la conducta prosocial e inhibiendo la conducta agresiva. Estos elementos han llevado a concluir, como lo plantean en su texto Mestre, et al (2002), que los individuos empáticos suelen ser menos agresivos y más propensos a manifestar comportamientos prosociales y de ayuda a otros, siendo la empatía opuesta al comportamiento agresivo (Chaux, 2012; Deceaty 2014; González-Blasco, etal., 2013).

Como se mencionó anteriormente, la empatía como proceso ha sido abordada no solo desde perspectivas teóricas sociales, filosóficas, socio-cognitivas, sino que ha venido siendo estudiada desde la neurociencia, de manera particular a partir de los hallazgos científicos logrados por Rizzollatti et al. (1996). Estudios realizados con primates (chimpancés, bonobos), así como estudios realizados con humanos han tenido como fin el explicar los comportamientos empáticos. A continuación se ofrece una breve descripción de los hallazgos en relación con las neuronas espejo y cómo a partir de dicho descubrimiento se ha logrado otra explicación de los procesos de interacción social, entre los cuales se encuentra tanto la comprensión de la empatía como la comprensión de comportamientos agresivos en los individuos.

\section{Perspectiva neurobiológica de la empatía}

El análisis de la empatía y el rol que ésta desempeña en las interacciones sociales desde un punto de vista biológico, lleva necesariamente al hallazgo fortuito realizado por Rizzolatti y sus colaboradores (1996), y descrito por lacoboni (2009), quienes 
al estudiar las neuronas premotoras en macacos, lograron descubrir la existencia de neuronas ubicadas en la zona prefrontal del cerebro, las que logran activarse con la sola observación por parte del primate del movimiento de otro sujeto, sin éste llegar a realizar ninguna acción. A partir de este momento, otros investigadores han profundizado en el evento, principalmente por la trascendencia que éste ha tenido en el estudio y comprensión de diversos comportamientos sociales de los seres humanos, incluidos no sólo aspectos que tienen que ver con los comportamientos empáticos, sino con la manifestación de comportamientos agresivos por imitación, o procesos relacionados con el lenguaje.

Es de tal trascendencia este hallazgo, no sólo para la neurociencia del comportamiento, sino en general para la psicología y ciencias afines, que Rachamandran (2000) citado por lacoboni (2009), expresa cómo el descubrimiento de las neuronas espejo es para la neurociencia lo que el ADN es para la biología, en lo que respecta a la explicación de las interacciones sociales. Teniendo en cuenta tal trascendencia, vale la pena explicar cuál es su papel en la manifestación de los comportamientos sociales, y de qué manera podría incidir en la empatía, específicamente en la población infantil y juvenil.

Como lo explica lacoboni (2009, p. 14)), “...existen grupos de células especiales en el cerebro que nos permiten entender a los demás [...];la activación en mi cerebro, al realizar otras personas mis propios movimientos me permite comprender sus intenciones...". Por lo tanto, según el autor, la activación de dichas células a nivel cerebral, van a permitir que los individuos puedan compartir emociones, experiencias, necesidades y metas, lo que finalmente termina incidiendo en la manera como los seres humanos desarrollan todas sus interacciones sociales. Al respecto, y de manera hipotética, valdría la pena formular como interrogante, si al lograr una mayor comprensión de motivos e intenciones entre los individuos se lograrían mayores niveles de comprensión en las interacciones sociales, y de manera consecuente, la construcción de ambientes de convivencia más pacíficos. Los hallazgos de Rizzolatti et al. (1996), lacoboni (2009) y en general los científicos de la neurociencia del comportamiento con sus hallazgos ofrecen una nueva alternativa para el futuro diseño y planteamiento de estrategias para el desarrollo de la empatía, y los comportamientos prosociales desde los ámbitos educativos, todo esto con miras a superar el problema de conflicto y agresión presente hoy en diversos contextos sociales.

Posterior a este hallazgo, en los últimos años se han venido desarrollando experiencias de carácter investigativo que buscan comprender el papel de las neuronas espejo en el mejoramiento de las interacciones sociales de los individuos. En este sentido, lacoboni (2009) cita los experimentos desarrollados por Ulf Dimberg, quien realizó mediciones de rostros felices y enfadados. Como lo explica el autor, existe a nivel cerebral un vínculo entre la zona donde se encuentran ubicadas las neuronas espejo y el sistema límbico, el cual está a cargo de las emociones; por lo tanto, 
en el desarrollo de su experimento, el investigador encontró que no solamente se activaban las neuronas espejo como reacción frente a los rostros que expresaban las emociones ya descritas, sino que también se activaba la zona límbica. Se lleva a cabo un circuito, en el cual interviene la expresión facial de la emoción como estímulo de las neuronas espejo; éstas a su vez estimulan la ínsula, ésta hace reaccionar al sistema límbico, el cual finalmente lleva a la experimentación de la emoción por parte del individuo que está recibiendo el estímulo inicial (lacoboni, 2009).

Desde el punto de vista biopsicológico, es importante hacer mención a las deficiencias en la función empática, las cuales se hacen evidentes en dos cuadros clínicos descritos por Koski y Sterck (2010) en su estudio comparativo de los componentes cognitivo y emocional de la empatía en humanos y chimpancés; las autoras hacen referencia a la capacidad empática limitada presente en las psicopatías, así como en el síndrome de espectro autista (ASD). En el primer cuadro, destacan la deficiencia en cuanto a la incapacidad del individuo de brindar una respuesta emocional frente a las reacciones de los otros, mientras que en el síndrome autista, las dificultades están presentes en el procesamiento de información desde lo cognitivo; tienen dificultad para reconocer las emociones en los otros, y este hecho incide en el reconocimiento de sus propias emociones.

A este punto de la reflexión, valdría la pena formular algunos interrogantes orientados a ligar los hallazgos de la neurociencia del comportamiento con escenarios escolares, preguntándonos a manera de hipótesis, si fuese posible desarrollar experiencias en el ámbito educativo que hicieran evidente la puesta en escena de la estimulación de las neuronas espejo mediante la exposición de situaciones, escenas, contextos, que permitan a niños, niñas y jóvenes observar situaciones de la vida cotidiana con miras a incidir en la vivencia y experiencia de las emociones de otros, es decir, fomentar la empatía, y generar así niveles de sensibilización y conciencia que aporten a la construcción de entornos sociales armónicos. El interrogante propuesto sirve de enlace para el abordaje de algunas experiencias de carácter investigativo llevadas a cabo en contextos educativos, a través de las cuales se puede verificar el papel que representa el desarrollo de procesos empáticos a lo largo del desarrollo.

\section{La empatía y su desarrollo: investigaciones empíricas}

Con respecto a la empatía y su evolución, Koski y Serck (2010) realizan una descripción paso a paso de los diferentes momentos a lo largo del desarrollo del niño y la manifestación de los comportamientos empáticos. Las investigadoras resaltan el papel del componente cognitivo de la empatía; es así como, al hablar de su desarrollo hacen referencia a cómo el procesamiento cognitivo del infante depende de la habilidad en él para construir representaciones mentales. Este hecho lleva a que se le brinde a la empatía y su componente cognitivo una verdadera manifestación del mismo hasta que el niño ha madurado suficientemente su capacidad para tomar 
perspectiva e identificar desde un punto de vista cognitivo, los estados emocionales del otro y diferenciarlos de los propios.

Desde otra perspectiva teórica respecto a la empatía, su tipología y evolución, se destaca la necesidad de retomar los postulados de Hoffman, citado por Otiz, M. et al. (1993) quien menciona los tipos de empatía disposicional y situacional, entendidas respectivamente, la primera como la tendencia a experimentar lo que sienten otros, mientras que la situacional hace referencia a dicha experiencia pero ante una situación concreta. En este sentido, investigadores han tratado de precisar el nivel predictivo de la empatía en la manifestación posterior de los comportamientos altruistas y de ayuda, respecto a los cuales se ha encontrado que existe una relación positiva.

Complementario a dicho planteamiento, Otiz, M. et al. (1993) insisten en la necesidad debrindar a los niños, niñas y adolescentes en formación entornos confiables y seguros, así como aprovechar diversas situaciones de la vida cotidiana que les permita sensibilizarse hacia sus sentimientos y necesidades, esto último, con miras a reforzar y potenciar las tendencias naturales de los niños a empatizar con el dolor del otro.

En la misma línea, Fuentes (1989) con su estudio ofrece un análisis evolutivo de la empatía y la ansiedad, como posibles variables mediadoras de los comportamientos de ayuda en niños y adolescentes. Estas dos investigaciones resaltan elementos que, para el presente trabajo de reflexión del proceso, son fundamentales por cuanto dejan ver la incidencia que tiene el fortalecimiento de la empatía entre la población infantil y juvenil para el mejoramiento en la calidad de las interacciones sociales de niños, niñas y jóvenes.

En relación con la empatía y la manera como llega a manifestarse en diferentes etapas del desarrollo, Garaigardobil y García (2006), en su estudio, hacen mención a las relaciones positivas que se han encontrado desde el punto de vista empírico entre los comportamientos prosociales entre niños y jóvenes y la empatía situacional. De la misma forma en el mismo estudio, citan algunas investigaciones que revelan la relación negativa existente entre empatía y agresión o entre la empatía y la manifestación de agresión verbal y física entre adolescentes.

Respecto a la evolución de la empatía, Cepeda (2004) en su estudio resalta la relación inversa existente entre agresión y empatía. En dicha investigación, a través de la aplicación de diferentes estrategias de medición, la autora logra determinar el tipo de relaciones que prevalecen entre las manifestaciones agresivas y los tipos de empatía manifestados por niños de cuarto de primaria, estableciendo mediciones puntuales en cuanto a los componentes cognitivo y afectivo de la empatía.

Entre los estudios más recientes que aborda la relación entre la empatía y la conducta prosocial, la agresividad, la autoeficacia y la responsabilidad personal y 
social de los estudiantes, se encuentra el trabajo desarrollado por Gutiérrez, Escartí y Pascual (2011), quienes a través de la medición de cada una de las variables psicosociales ya mencionadas, en 822 estudiantes de 8 a 15 años, pertenecientes a grado 11 en centros educativos de Valencia, España, logran demostrar con su modelo, cómo una trayectoria de vida psicológicamente saludable es el producto de la combinación del aprendizaje de habilidades y competencias sociales útiles para la vida, junto con unas condiciones que brinden apoyo, cuidado y seguridad. Los autores resaltan el papel de la empatía dentro de dicho proceso, y la describen como fundamental para el desarrollo psicológico/emocional positivo del individuo. De igual manera, a lo largo de su estudio resaltan la necesidad de fomentar en los niños, niñas y adolescentes comportamientos de empatía y autocontrol, comportamiento de apoyo y ayuda a los otros, así como fomentar en los estudiantes la capacidad para identificar los sentimientos y emociones de las personas que les rodean, todo esto con miras a promover la responsabilidad social y personal en la población infantil y juvenil.

Otro estudio reciente que amerita mención, es el descrito por Guillen, J. (2014), quien hace referencia a la aplicación del concepto de neuronas espejo en el aula, a través de la realización de acciones por parte del maestro que incidan en la cognición social de los estudiantes. Según el autor, el clima emocional de aula depende del profesor. Es así, como la actitud que manifieste el maestro al momento de ingresar a la clase, determinará de alguna manera, los comportamientos y actitudes de los estudiantes, si se tiene en cuenta la influencia de este sistema neuronal en quienes reciben estímulos sociales, bien sean éstos de carácter positivo o negativo. De igual manera, destaca que esa comprensión empática por parte de quien recibe la información emocional (en este caso el estudiante), le permitirá identificar y comprender el estado emocional del maestro, y la influencia de este proceso será determinante en la postura del estudiante frente al desarrollo de su clase, y por ende del aprendizaje logrado. Todos estos hallazgos en el campo de las emociones, los sentimientos y las interacciones sociales en el ámbito educativo, resaltan la necesidad de fortalecer la investigación frente a este tema, si se desea propender por la construcción de entornos escolares para los niños del mañana.

\section{¿Cómo crear una cultura prosocial y de ayuda mediante el desarrollo de la empatía?}

Responder a este interrogante debe ser propósito de un estudio más profundo y de mayor envergadura, sin embargo, vale la pena formularlo si se quieren contemplar los aspectos sociales, culturales y familiares que acorde con los estudios realizados hasta el momento, reflejan poseer un vínculo estrecho con la potencialización de manifestaciones empáticas en niños, niñas y adolescentes. En este sentido, Otiz M. et al. (1993), en su estudio, destacan la importancia que tiene el que tanto niños como niñas establezcan niveles de empatía con quien se encuentra en posición de 
víctima, para la manifestación de comportamientos prosociales y altruistas. De igual manera, en el mismo estudio se encuentra una relación positiva entre la presencia de comportamientos prosociales en los hijos de padres que han manifestado comportamientos prosociales. Estos aspectos destacan la importancia del fomento de la empatía en diversos contextos de formación e interacción social de los niños y jóvenes.

\section{Comentarios finales}

Las diferentes perspectivas teóricas, así como los trabajos empíricos descritos a lo largo del presente documento, son un Ilamado a los investigadores de las ciencias humanas y sociales, así como a quienes se encuentran a cargo de procesos educativos y de formación de las futuras generaciones, a desarrollar experiencias de prevención e intervención que favorezcan y aporten a la construcción de ambientes de interacción sanos y armónicos desde el punto de vista biofísico, psicológico y social.

El desarrollo de experiencias educativas y pedagógicas orientadas al fomento de procesos empáticos que permitan la formación de niños, niñas y jóvenes sensibles frente al dolor del otro, capaces de comprender sus propias emociones y sentimientos, y consecuentemente estar en capacidad de entender sus experiencias emocionales, podría ser un primer paso en la búsqueda de entornos escolares más gratos y que verdaderamente aporten a la formación de individuos que a futuro puedan ser constructores de sociedad.

Este puede ser un llamado para los educadores, psicopedagogos y en general profesionales de la educación, que orienten el diseño de estrategias pedagógicas, educativas y didácticas al fortalecimiento de procesos empáticos, con miras primordialmente a generar procesos de sensibilización desde los primeros años de formación, en la búsqueda por la construcción de entornos de interacción más amables, que permitan superar el conflicto y la agresión presentes hoy.

A partir de este planteamiento y desde un punto de vista empírico, las investigaciones abordadas y descritas brindan luces respecto a posibles maneras de fomentar la empatía en los entornos escolares. A manera de conclusión, una de ellas podría estar orientada al diseño de propuestas de prevención dirigidas al fomento de procesos empáticos, de carácter transversal (cobertura a todos los niveles de formación: preescolar, básica y media), con la implicación en el proceso de toda la comunidad educativa (estudiantes, maestros y padres de familia). Si se tiene en cuenta ese factor multidimensional que posee la empatía como proceso, en el cual intervienen aspectos neuropsicológicos, sociales y cognitivos, el diseño de experiencias de aula que aborden los tres aspectos, podría favorecer dicho desarrollo. 


\section{Referencias}

Cepeda, A. (2004). La relación inversa entre empatía y agresión: precisiones conceptuales y metodológicas. Un estudio con niñas de cuarto de primaria. Tesis de Psicología no publicada. Universidad de Los Andes, Bogotá, Colombia. Recuperada de http://biblioteca. uniandes.edu.co/acepto42.php?id=00003766

Chaux, E. (2004). Agresión reactiva, agresión instrumental y el ciclo de la violencia. Revista de estudios sociales. 15: 47-58

Chaux, E. (2012). Educación, convivencia y agresión escolar. Bogotá: Alfaguara, pp. 250

Deceaty, Jean. (2014). La contribución del sistema de las neuronas espejo a la empatía. Ciclo de Conferencias en Intramed. Simposio Internacional de Neurociencias Cognitivas y Psiquiatría. Recuperado de http://www.intramed.net/contenidover.asp?contenidolD=622 $72 \#$ comentarios

Eisenberg, N. y Strayer, J (1992). La empatía y su desarrollo. Bilbao: Editores Desclé de Brouwer.

Feshbach, N. (1983). Learning to care: a positive approach to child training and discipline. Journal of Clinical Child Psychology. 12 (3): 266-271.

Fuentes, M. J. (1989). Análisis evolutivo de la empatía y la ansiedad como variables mediadoras de los comportamientos de ayuda. Infancia y Aprendizaje: 48: 65-78.

Garaigardobil, M., García, P. (2006). Empatía en niños de 10 a 12 años. Psicothema.18 (2): 180-186.

García, E., González, J.. Maestú, F. (2011). Neuronas espejo y la teoría de la mente en la explicación de la empatía. Ansiedad y Stress. 17 (2-3): 265-279.

González-Blasco, P. et al. (2013). Educar las emociones para promover la formación ética., Persona y Bioética.. (17)1: 28-48.

Gutiérrez, M., Escartí, A., Pascual, C. (2011). Relaciones entre empatía, conducta prosocial, agresividad, autoeficacia y responsabilidad personal y social de los escolares. Psicothema. 23(1): 13-19.

lacoboni, M. (2009). Las neuronas espejo. Empatía, neuropolítica, autismo, imitación o de cómo entendemos a los otros. Recuperado de http://books.google.com.co/books?id=C5RH gLTPmlwC\&pg=PA5\&hl=es\&source=gbs_selected_pages\&cad $=3 \# \mathrm{v}=$ onepage \&q\&f=false

Koski, S., Sterck, E. (2010). Empathic chimpanzees: A proposal of the level of emotional and cognitive processing in chimpanzee empathy. European Journal of Developmental Psychology. (7) 1: 38-66.

Mestre, M., Samper, P., Frías, M. (2002). Procesos cognitivos y emocionales predictores de la conducta prosocial y agresiva: la empatía como factor modulador. Psicothema. 14(2): 227-232.

Moya-Albio, L., Herrero, N., Bernal, C. (2010). Bases Neuronales de la empatía. Revista de Neurología. 50 (2): 89-100. 
Otiz, Ma. J. et al. (1993). Algunos predictores de la conducta prosocial-altruista en la infancia: empatía, toma de perspectiva, apego, modelos parentales, disciplina familiar e imagen del ser humano. Revista de psicología social. 8 (1): 83-98.

Rizzolatti, G., Fadiga, L., Gallese, V., Fogassi, L. (1996). Premotor cortex and the recognition of motor actions. Cognitive brain research. 3: 131-141 Recuperado de web.mit.edu/gorlins/ Public/Motor\%20System/Rizzolatti_1996.pdf

Salmivalli, C, et al. (1996). Bullying as a group process: Participant roles and their relations to social status within the group. Aggressive Behavior. 22: 1-15.

Spelling, M. (sf). The effect of an empathy training program on aggression in elementary age children. Recuperado de www.etbu.edu/export/.../honorsProgram/.../Maria_SpellingsFall_2007.pdf

Recibido: 7 mayo 2013

Aceptado: 9 septiembre 2013

Como citar:

Galvis, R.I. (2014) Las neuronas espejo y el desarrollo de la empatía frente a la agresión y el conflicto en la escuela. Praxis Pedagógica 15, 43-53 